\title{
An Approximate Expression for Gamma-Ray Degradation Spectra
}

\author{
U. Fano and Ann T. Nelms
}

\begin{abstract}
The equation for gamma-ray degradation spectra is reduced to a form suitable for a simple approximation. The approximation is valid in a wavelength range between two Compton wavelengths larger than the wavelength of the source and the region where photoelectric absorption becomes very intense; this range is wide for low ? materials. Input data are provided for application to various materials and source energies. The results of sample calculations are shown and compared with those obtained by standard numerical calculation.
\end{abstract}

In the course of gamma-ray penetration, the spectrum of multiply scattered, degraded, secondaries approaches a shape that depends only on the properties of the medium. ${ }^{1}$ This limiting spectrum is that which arises when a gamma-ray source is distributed uniformly throughout a medium and the only relevant factor is the accumulation at each point of secondaries of various energies. When degradation spectra were first calculated numerically, ${ }^{2} \mathrm{M}$. H. Johnson pointed out, in a private discussion, that the main features of the degradation spectrum at low energies are determined by very simple arguments. A formalism of degradation theory, starting from this remark and from the corresponding neutron theory, ${ }^{3}$ was developed later. ${ }^{4}$ But this theory was not applied in actual gamma-ray calculations, because these were being carried out routinely by direct numerical computation. ${ }^{5}$ Lately, the extension of calculations to degraded photon energies $\approx 20$ kev has made it desirable to try to evaluate the simple approximate analytical formulas and compare the results with those obtained by numerical integration of the basic equations. These low photon energies are of interest, e. g., in $\mathrm{LiH}$. This paper contains a description of the procedure, beginning with the qualitative analysis, together with a discussion of the results of the comparison.

If successive steps of degradation by Compton scattering, characterized by wavelength shifts $\xi=\lambda-\lambda^{\prime}$, have approximately equal statistical distribution (see footnote 4 ) with a mean $\langle\xi\rangle$, the probability for each photon to attain a wavelength between $\lambda$ and $\lambda+\Delta \lambda$ at the end of one of the steps of degradation is $\Delta \lambda /\langle\xi\rangle .^{6}$ If the photon does "land" in this interval, it will "spend" there a mean free path $1 / \mu_{\text {scatt }}(\lambda)$, where $\mu_{\text {seat }}$ is the probability of scattering per unit pathlength and coincides with the total $\mu(\lambda)$ when absorption is disregarded. For a source strength of $S$ photons per unit volume and

\footnotetext{
1 See, e. g., U. Fano, Nucleonics 11, Nos. 8 and 9, 55 (1953).

2 P. R. Karr and J. C. Lamkin, Phys. Rev. 76, 1843 (1949)

${ }^{3}$ See, e. g., R. Marshak, Rev. Mod. Phys. 19, 185 (1947), and in particular, G. Placzek, Phys. Rev. 69, 423 (1946).

4 Placzek, Phys. Rev. 69, $423(1946)$.
U. Fano, Phys. Rev. 92, 328 (1953)

5 Calculations of penetration by the moment method, as given by H. Goldstein and J. E. Wilkins, Jr., NYO Report 3075 (1954), include, under the name of "zero-th moment", the spectrum discussed here

"zero-th moment", the spectrum discussed here.
"This problem is similar to the determination of the probability that a pedes${ }^{6}$ This problem is similar to the determination of the probability that a pedes-
trian will step on a line drawn across a sidewalk. The probability equals the
} length of the pedestrian's foot divided by his mean step length. unit time, we thus expect at each point a flux of photons in the assigned wavelength range

$$
I(\lambda) \Delta \lambda=S[1 / \mu(\lambda)](\Delta \lambda /\langle\xi\rangle)
$$

per unit area and per unit time. This approximate spectral distribution is expressed in the scale of wavelenoths because it is in this scale that the statistical distribution of successive steps of degradation is more nearly a constant.

Equation (1) is subject to three main limitations: (a) It does not hold at photon energies $\geq 500 \mathrm{kev}$, where the distribution of degradation steps varies rapidly from step to step; (b) it represents the spectrum for a "steady state" of degration, which does not hold for photons that have been scattered only a very few times because their spectral distribution depends on the source wavelength ("transient" effect) (see footnote 4); (c) it does not take into account outright photon absorption, by photoelectric effect. The effect of absorption can easily be included in (1) as a progressive decay of intensity as $\lambda$ increases, provided the probability of absorption at each step is small.

Equation (1) is contained as a special case in the solution of degradation problems by successive approximations (see footnote 4). In this solution the degradation spectrum is resolved into steady state and transient components. A numerical calculation of the first few terms of this solution for the X-ray degradation would be possible, but it was found more convenient to proceed as in the analogous electron degradation problem. ${ }^{7}$ One starts from an integral form of the degradation equation, namely

$$
\int_{\lambda_{0}}^{\lambda} d \lambda^{\prime} I\left(\lambda^{\prime}\right)\left[\mu_{\text {abs }}\left(\lambda^{\prime}\right)+\int_{\lambda}^{\lambda^{\prime}+2} k\left(\lambda^{\prime}, \lambda^{\prime \prime}\right) d \lambda^{\prime \prime}\right]=S
$$

which represents a sort of "balance sheet" of photons in the entire wavelength range from $\lambda_{0}$ to $\lambda$. The source injects $S$ photons per unit volume and unit time at $\lambda_{0}$. Of these, $\int_{\lambda_{0}}^{\lambda} d \lambda^{\prime} I\left(\lambda^{\prime}\right) \mu_{\text {abs }}\left(\lambda^{\prime}\right)$ are lost through outright absorption, and the remainder by Compton scattering with final wavelength $\lambda^{\prime \prime}>\lambda$,

7 L. V. Spencer and U. Fano, Phys. Rev. 93, 1172 (1954). 
where $k\left(\lambda^{\prime}, \lambda^{\prime \prime}\right) d \lambda^{\prime \prime}$ is the probability per unit pathlength that a photon of wavelength $\lambda^{\prime}$ be scattered to a final wavelength between $\lambda^{\prime \prime}$ and $\lambda^{\prime \prime}+d \lambda^{\prime \prime}$. Note that the wavelengths are expressed in Compton units of $h / m c=2.4 \times 10^{-10} \mathrm{~cm}$.

Equation (2) should now be transformed so that it displays an approximate solution similar to (1) and so that the necessary corrections can be easily estimated and then calculated either by successive approximation or by numerical solution of the new equation. To minimize the difficulties (a) and (b) indicated above, we restrict our discussion to wavelengths $\lambda>\lambda_{0}+2$; thus no photon from the source can reach $\lambda$ in a single Compton scattering, because $k\left(\lambda^{\prime}, \lambda^{\prime \prime}\right)=0$ for $\lambda^{\prime \prime}>\lambda^{\prime}+2$. As a first step we transform the Compton scattering term in (2), by setting $\lambda^{\prime \prime}=\lambda^{\prime}+\xi$ and $\lambda^{\prime}=\lambda-\eta$, and find

$$
\begin{aligned}
\int_{\lambda_{0}}^{\lambda} d \lambda^{\prime} I\left(\lambda^{\prime}\right) \int_{\lambda}^{\lambda^{\prime}+2} k\left(\lambda^{\prime}, \lambda^{\prime \prime}\right) d \lambda^{\prime \prime} \\
\quad=\int_{0}^{2} d \eta I(\lambda-\eta) \int_{\eta}^{2} k(\lambda-\eta, \lambda-\eta+\xi) d \xi \\
=\int_{0}^{2} d \xi \int_{0}^{\xi} d \eta I(\lambda-\eta) k(\lambda-\eta, \lambda-\eta+\xi) .
\end{aligned}
$$

Because the procedure that is being developed is expected to apply when $I$ and $k$ vary slowly with $\eta$, it is convenient to represent (3) in a form suited to expansion in powers of $\eta$. This is done by an operator symbol based on the power expansion formula

$$
\begin{aligned}
f(\lambda-\eta)=f(\lambda)-\eta \frac{\partial}{\partial \lambda} f(\lambda)+\frac{1}{2} \eta^{2} \frac{\partial^{2}}{\partial \lambda^{2}} f(\lambda) & \\
& +\ldots=e^{-\eta \frac{\partial}{\partial \lambda}} f(\lambda) .
\end{aligned}
$$

The integrand on the right of (3) can therefore be indicated in the form

$$
I(\lambda-\eta) k(\lambda-\eta, \lambda-\eta+\xi)=e^{-\eta \frac{\partial}{\partial \lambda}} I(\lambda) k(\lambda, \lambda+\xi) .
$$

We consider in particular the value of (3) for $\eta$ set equal to zero in the exponent of (5). This value is

$$
I(\lambda) \int_{0}^{2} d \xi \xi k(\lambda, \lambda+\xi)=I(\lambda) M_{1}(\lambda) .
$$

The function $M_{1}(\lambda)$ defined here is the first moment of the wavelength shift and may be expressed as $\mu_{\text {seat }}\langle\xi\rangle$, which coincides with the denominator of (1). We add the right side of (6) to the expression (3) and subtract its left side from (3), utilize (5) and combine the result with the degradation equation (2), which becomes

$$
\begin{aligned}
I(\lambda) M_{1}(\lambda) & +\int_{\lambda_{0}}^{\lambda} d \lambda^{\prime} I\left(\lambda^{\prime}\right) \mu_{\text {abs }}\left(\lambda^{\prime}\right) \\
& +\int_{0}^{2} d \xi \int_{0}^{\xi} d \eta\left(e^{-\eta \frac{\partial}{\partial \lambda}}-1\right) I(\lambda) k(\lambda, \lambda+\xi)=S .
\end{aligned}
$$

The solution (1) is thereby obtained if the integrals in this equation can be disregarded by assuming $\mu_{\text {abs }} \approx 0$, and $\eta \partial / \partial \lambda \approx 0$ in the exponent.

Consider now the absorption, represented by the first integral in (7), taking into account that $I M_{1}$ should be effectively constant in the absence of absorption. The absorption term is then written

$$
\int_{\lambda_{0}}^{\lambda} d \lambda^{\prime} \mu_{\mathrm{abs}}\left(\lambda^{\prime}\right) I\left(\lambda^{\prime}\right)=\int_{\lambda_{0}}^{\lambda} d \lambda^{\prime} \beta\left(\lambda^{\prime}\right)\left[I\left(\lambda^{\prime}\right) M_{1}\left(\lambda^{\prime}\right)\right],
$$

showing that $I M_{1}$ is effectively reduced, as $\lambda$ increases, by a fraction

$$
\beta(\lambda) \Delta \lambda=\frac{\mu_{\text {abs }}(\lambda)}{M_{1}(\lambda)} \Delta \lambda=\frac{\mu_{\text {abs }}(\lambda)}{\mu_{\text {scatt }}(\lambda)} \frac{\Delta \lambda}{\langle\xi\rangle}
$$

in every small interval $\Delta \lambda$. Notice that this fraction has the dimensions of a pure number. This absorption effect, taken by itself, would cause $I M_{1}$ to decay, as $\lambda$ increases, according to $\exp \left[-\int_{\lambda_{0}}^{\lambda} \beta\left(\lambda^{\prime}\right) d \lambda^{\prime}\right]$. Accordingly we replace the spectral distribution $I(\lambda)$ with a new unknown $w(\lambda) \approx 1$ defined by the expression

$$
I(\lambda)=\frac{S}{M_{1}(\lambda)} e^{-\int_{\lambda_{0}}^{\lambda} \beta\left(\lambda^{\prime}\right) d \lambda^{\prime}} w(\lambda) .
$$

When this expression for $I(\lambda)$ is substituted in (7), the source strength $S$ drops out as a common factor. In the first term we use the identity

$$
e^{-\int_{\lambda_{0}}^{\lambda^{\prime}} \beta\left(\lambda^{\prime}\right) d \lambda^{\prime}}=1-\int_{\lambda_{0}}^{\lambda} d \lambda^{\prime} \beta\left(\lambda^{\prime}\right) e^{-\int_{\lambda_{0}}^{\lambda^{\prime}} \beta\left(\lambda^{\prime \prime}\right) d \lambda^{\prime \prime}},
$$

so that the second term from this expression may be combined with the absorption term in (7). In the second integral of $(7)$ the term $\exp \left[-\int_{\lambda_{0}}^{\lambda} \beta\left(\lambda^{\prime}\right) d \lambda^{\prime}\right]$ may be factored out utilizing the operator formula

$$
e^{-\eta \frac{\partial}{\partial \lambda}} e^{-\int_{\lambda_{0}}^{\lambda} \beta\left(\lambda^{\prime}\right) d \lambda^{\prime}}=e^{-\int_{\lambda_{0}}^{\lambda} \beta\left(\lambda^{\prime}\right) d \lambda^{\prime}} e^{-\eta\left[\frac{\partial}{\partial \lambda}-\beta(\lambda)\right]} ;
$$

after factoring, (11) is applied again. Equation (7) becomes now

$$
\begin{gathered}
w(\lambda)+\int_{\lambda_{0}}^{\lambda} d \lambda^{\prime} \beta\left(\lambda^{\prime}\right) e^{-\int_{\lambda_{0}}^{\lambda^{\prime}} \beta\left(\lambda^{\prime \prime}\right) d \lambda^{\prime \prime}}\left\{w\left(\lambda^{\prime}\right)-\int_{0}^{\xi} d \xi \int_{0}^{\xi} d \eta\right. \\
\left.e^{-\eta\left[\frac{\partial}{\partial \lambda}-\beta(\lambda)\right]} \frac{k(\lambda, \lambda+\xi)}{M_{1}(\lambda)} w(\lambda)\right\}+\int_{0}^{2} d \xi \int_{0}^{\xi} d \eta \\
\left(e^{-\eta\left[\partial \partial^{\partial}-\beta(\lambda)\right]}-1\right) \frac{\kappa(\lambda, \lambda+\xi)}{M_{1}(\lambda)} w(\lambda)=1 .
\end{gathered}
$$

This equation is fully equivalent to the initial eq (2), for $\lambda>\lambda_{0}+2$. Its zero-order approximation solution is $w(\lambda)=1$, obtained by disregarding all the 
integrals. To obtain a better approximation, the integrals may be evaluated by whatever procedure appears expedient. We proceed here by expanding into powers of the operator $\partial / \partial \lambda$ and of the rate of absorption $\beta$, to first order only. The integral over $\lambda^{\prime}$ yields no contribution to first order, because of the factor $\beta\left(\lambda^{\prime}\right)$ and because the factor contained in the curly brackets vanishes in zero-th order. Where the zero order approximation \{\}$=0$ is poor, for $\lambda<\lambda_{0}+2, \beta\left(\lambda^{\prime}\right)$ is very small for most materials. The second integral is evaluated to first order by writing

$$
\begin{aligned}
& \left.\int_{0}^{\xi} d \eta\left(e^{-\eta\left[\frac{\partial}{\partial \lambda}-\beta(\lambda)\right.}\right]-1\right) \approx-\int_{0}^{\xi} d \eta \eta\left[\frac{\partial}{\partial \lambda}-\beta(\lambda)\right] \\
= & -\frac{1}{2} \xi^{2}\left[\frac{\partial}{\partial \lambda}-\beta(\lambda)\right]
\end{aligned}
$$

and $w(\lambda) \approx 1$. Integration over $\xi$ yields the first order estimate of the second integral

$$
-\left[\frac{\partial}{\partial \lambda}-\beta(\lambda)\right] \frac{1}{2} \frac{M_{2}(\lambda)}{M_{1}(\lambda)}
$$

where $M_{2}(\lambda)=\int_{0}^{2} d \xi \xi^{2} k(\lambda, \lambda+\xi)$ indicates the second moment of the wavelength shitt $\xi$. Thus we find

$$
w(\lambda)=1+\frac{\partial}{\partial \lambda} \frac{1}{2} \frac{M_{2}(\lambda)}{M_{1}(\lambda)}-\beta(\lambda) \frac{1}{2} \frac{M_{2}(\lambda)}{M_{1}(\lambda)}+\ldots
$$

and

$$
\begin{aligned}
I(\lambda)=\frac{S}{M_{1}(\lambda)} e^{-\int_{\mathrm{\chi}_{0}}^{\lambda} \beta\left(\lambda^{\prime}\right) d \lambda^{\prime}} & {\left[1+\frac{\partial}{\partial \lambda} \frac{1}{2} \frac{M_{2}(\lambda)}{M_{1}(\lambda)}\right.} \\
& \left.-\beta(\lambda) \frac{1}{2} \frac{M_{2}(\lambda)}{M_{1}(\lambda)}+\cdots\right] .
\end{aligned}
$$

The function (1/2) $M_{2} / M_{1}$ was determined analytically using the Klein-Nishina formula for Compton scattering. A graphical differentiation was used to obtain $(\partial / \partial \lambda)(1 / 2) M_{2} / M_{1}$. These data, which are the same for all materials, are given in figure 1 . The absorption exponent $\int_{0}^{\lambda} d \lambda^{\prime} \beta\left(\lambda^{\prime}\right)$, where only photoelectric absorption is included in the tabulations of $\beta\left(\lambda^{\prime}\right)$, is given for a few materials in figure 2 . The computation of $\beta\left(\lambda^{\prime}\right)$ utilized total attenuation coefficients from G. Grodstein ${ }^{8}$ and the total Compton cross section from the Klein-Nishina formula. The integration was carried out numerically. Figure 3 gives values of $M_{1}^{-1}(\lambda)$. The solid curve is a dimensionless plot of the quantity $\left[\left(\mu_{\text {Compton }} / \mu_{\text {Thompson }}\right)\langle\xi\rangle\right]^{-1}$, which is independent of atomic number. For a particular material, the nomograph on the left permits the conversion to units of $\mathrm{g} / \mathrm{cm}^{2}$. If the source yields $S$ photons $\mathrm{g}^{-1} \mathrm{sec}^{-1}, S / M_{1}(\lambda)$ is in units $\mathrm{cm}^{-2} \mathrm{sec}^{-1}(\mathrm{~h} / \mathrm{mc})^{-1}$.

With the data from figures 1 to 3 the zero-th order expression may be calculated, and the importance of the first order correction may be evaluated

\footnotetext{
8 G. W. Grodstein, NBS Circular 583 (1957).
}

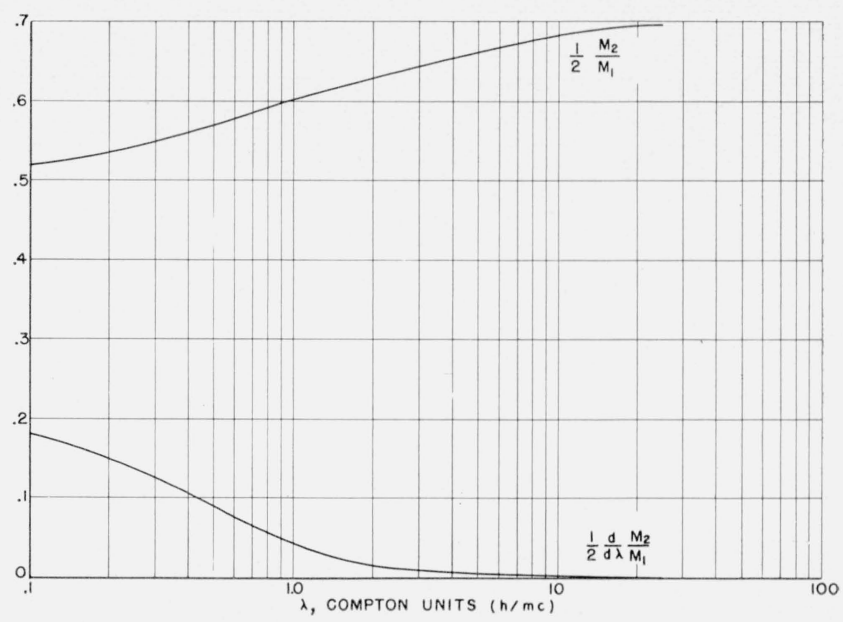

FiguRE 1. Graph of corrective parameters in eq $1 \%$.

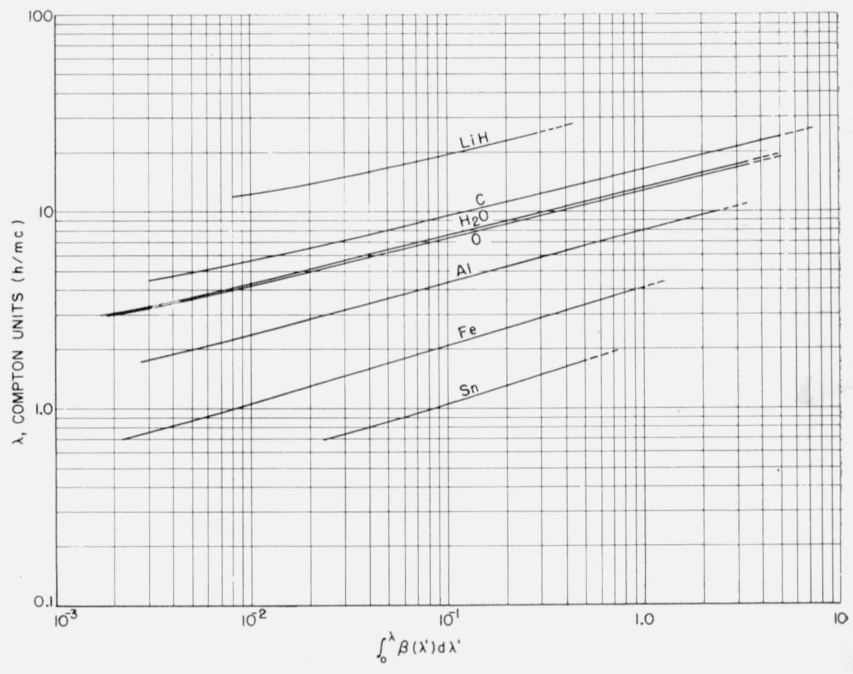

Figure 2. The absorption exponent $\int_{0}^{\lambda} \beta\left(\lambda^{\prime}\right) d \lambda^{\prime}$ for several materials.

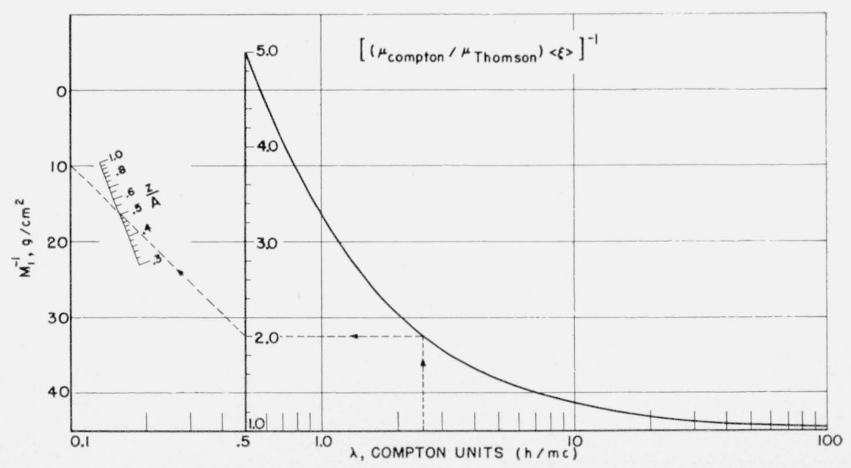

Figure 3. Spectral distribution of photon track length. 


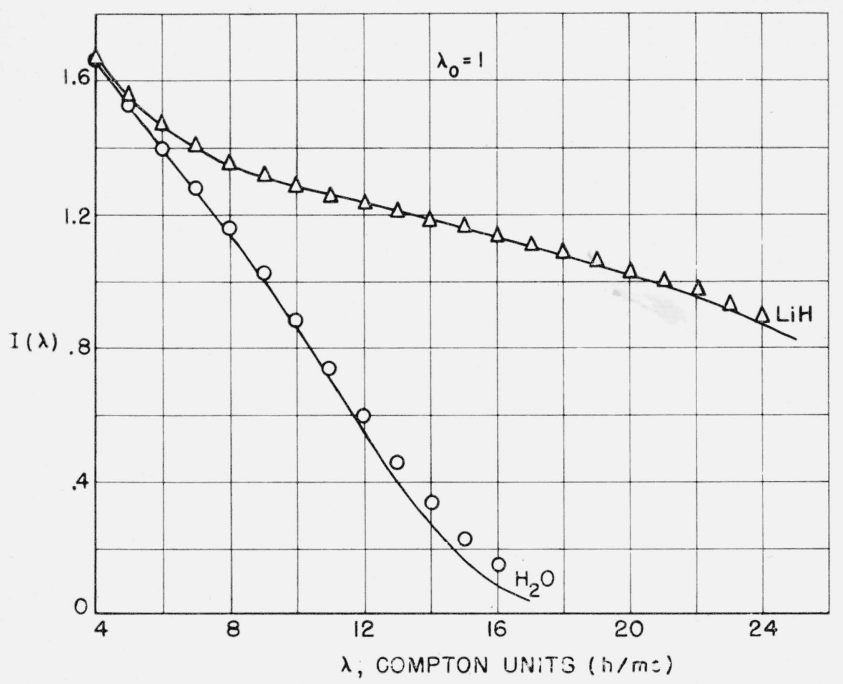

Figure 4. Degradation spectra for $\mathrm{LiH}$ and $\mathrm{H}_{2} \mathrm{O}$. Approximation, eq (17); $\bigcirc, \Delta$, exact numerical integration.

readily. This has been done for two cases, namely gamma rays with initial wavelength $\lambda_{0}=1$ in $\mathrm{LiH}$ and $\mathrm{H}_{2} \mathrm{O}$, the source strength $S$ being unity. The results are the curves in figure 4 . The points in the figure show the results of direct numerical integration. The agreement is well within the expected accuracy of the approximation. The departures at increasing wavelength reflect the increasing strength of photoabsorption, which leads eventually to a breakdown of the validity of the assumption that the absorption changes little over a wavelength range $\langle\xi\rangle \approx 1$.

Of general interest is the absorption "cut-off" of the spectra. This point, which may be characterized by a parameter $\lambda_{z}$ satisfying the relation $\int_{0}^{\lambda} z\left(\lambda^{\prime}\right)$

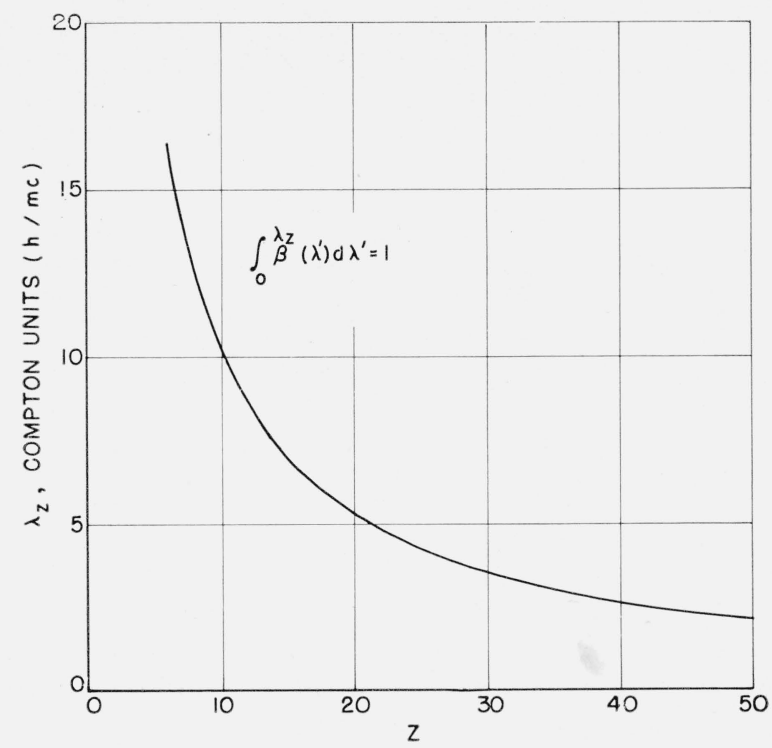

Figure 5. Absorption "cut-off" of the spectral distribution.

$d \lambda^{\prime}=1$. It is the wavelength or energy at which the absorption reduces sharply the spectral distribution. Figure 5 gives a curve of $\lambda_{Z}$ versus the atomic number $Z$. Again, the values for $\beta\left(\lambda^{\prime}\right)$ take into account only photoelectric absorption.

The authors thank L. V. Spencer and M. Berger for many helpful discussions, and J. C. Lamkin, who contributed the results of the direct numerical integration.

Washington, March 12, 1957. 